\title{
Vigilância epidemiológica da leishmaniose visceral: análise de indicadores e fatores ambientais associados
}

\author{
Epidemiological surveillance of visceral leishmaniasis: analysis of indicators and correlated \\ environmental factors
}
Vigilancia epidemiológica de leishmaniose visceral: análisis de los indicadores y factores ambientales asociados

Karliane de Araújo Lima Uchôa ${ }^{1}$, Baldomero Antonio Kato da Silva ${ }^{1 *}$, Ana Rachel Oliveira de Andrade $^{2}$, Karina Oliveira Drumond ${ }^{3}$.

\section{RESUMO}

Objetivo: analisar os indicadores epidemiológicos da Leishmaniose Visceral (LV) em Parnaíba-PI no período de 2010 a 2014. Métodos: foram incluídos 45 casos novos de Leishmaniose Visceral Humana (LVH) autóctone, residentes em Parnaíba e população canina de 2787 cães. Os dados foram obtidos do Sistema de Informação de Agravos de Notificação (SINAN), Sistema de Informação sobre Mortalidade (SIM), Instituto Brasileiro de Geografia de Estatística (IBGE), inquérito canino do Centro de Controle de Zoonoses (CCZ) e questionários semiestruturados. Resultados: observou-se média de nove casos de LVH nos últimos cinco anos, classificando-se como área de transmissão intensa para LV. Ocorreu registro de LVH em todos os meses do ano, com maior número de notificações nos meses de junho $(n=8)$ e outubro $(n=8)$. A soroprevalência canina foi de $16 \%$, com variação entre $8 \%$ a $42 \%$ durante o período, ascendendo a partir de 2013, com razão de 1:10 de casos humanos em relação aos caninos. Conclusão: os resultados oferecem subsídios para planejamento estratégico no controle da LV, propondo focalizar intervenções nas áreas estratificadas, incrementando a efetividade das medidas de controle na região.

Palavras-chave: Doenças negligenciadas, Monitoramento epidemiológico, Prevenção \& controle.

\begin{abstract}
Objective: epidemiological data analysis of Visceral Leishmaniasis (VL) in Parnaíba - PI over the period 2010 to 2014. Methods: It has been included 45 new cases of autochthonous Human Visceral Leishmaniasis (HVL) residing in the city of Parnaíba-PI and canine population of 2787 dogs. Data were obtained from the Information System for Notification of Complaints (SINAN), Mortality Information System (SIM), Brazilian Institute of Geography and Statistics (IBGE), stray canine survey templates from the Zoonoses Control Center (CCZ) and Semi-structured interviews. Results: observed an average of nine HVL cases in the last five years, being classified as an intense transmission area for HVL. There were HVL monthly registers throughout the year, with the highest notified case numbers in the months of June $(n=8)$ and October $(n=8)$. Canine seroprevalence was of $16 \%$, ranging from $8 \%$ to $42 \%$ during the analyzed period, with ascendance start in 2013 and presented a ratio of 1:10 of human cases in relation to canine cases. Conclusion: the results provided in this study offers strategic planning for VL control subsidies, proposing focused interventions in the stratified areas increasing the effectiveness of measure controls in the region.
\end{abstract}

Keywords: Neglected diseases, Epidemiological monitoring, Prevention \& Control.

1 Programa de Pós-Graduação em Ciências Biomédicas, Universidade Federal do Delta do Parnaíba (UFDPar), Parnaíba - PI, *E-mail: ftbaldock@uol.com.br

2 Faculdade de Medicina, Instituto de Educação Superior do Vale do Parnaíba II (ESVAP), Parnaíba - PI

${ }^{3}$ Curso de Biomedicina, Universidade Federal do Delta do Parnaíba (UFDPar), Parnaíba - PI 


\section{RESUMEN}

Objetivo: analizar los indicadores epidemiológicos de la Leishmaniasis Visceral (LV) en Parnaíba-PI en el período del 2010 al 2014. Métodos: fueron incluidos 45 casos nuevos de Leishmaniasis Visceral Humana (LVH) autóctona, residentes en Parnaíba y población canina de 2787 perros. Los datos fueron obtenidos a partir del Sistema de Información para la Notificación de Quejas (SINAN), Sistema de Información de Mortalidad (SIM), Instituto Brasileño de Geografía y Estadística (IBGE), planilla sobre encuesta canina del Centro de Control de Zoonosis (CCZ) y cuestionarios semiestructurados. Resultados: se observó un promedio de nueve casos de LVH en los últimos cinco años, clasificándose como área de transmisión intensa para LV. Ocurrió un registro de LVH en todos los meses del año, con mayor número de notificaciones en los meses de junio $(n=8)$ y octubre $(n=8)$. La seroprevalencia canina fue del $16 \%$, con variación entre el $8 \%$ al 42\% durante el período analizado, con ascendencia a partir del 2013 con una razón del 1:10 de casos humanos en relación a casos caninos. Conclusión: los resultados obtenidos ofrecen subsidios para planeamiento estratégico en el control de la LV, proponiendo enfocar las intervenciones en las áreas estratificadas, incrementando la efectividad de las medidas de control en la región.

Palabras-clave: Enfermedades olvidadas, Monitoreo epidemiológico, Prevención \& control.

\section{INTRODUÇÃO}

A Leishmaniose Visceral (LV) é uma das doenças mais negligenciadas no mundo, atingindo grande parte da população de baixa renda, com estimativa de 200 a 400 mil novos casos a cada ano. A continuidade desse agravo está diretamente relacionada com a pobreza, os fatores sociais, ambientais e climáticos, o sistema imunológico deficiente e a falta de recursos (WHO, 2016).

No período de 2010 a 2013, a média do coeficiente de incidência LV no Brasil, na região nordeste, no Estado do Piauí e na cidade de Parnaíba foi, respectivamente, de 1,75/100.000 habitantes (hab.), 3/100.000 hab., 5,7/100.000 hab. e 6,5/100.000. A elevada incidência de LV em Parnaíba-PI, que mostra o alto risco da população parnaibana adoecer por esse agravo, determina necessidade premente de análise da vigilância epidemiológica (BRASIL, 2014).

A vigilância epidemiológica é uma das ações prioritárias do programa nacional de controle da LV para reduzir as taxas de letalidade e do grau de morbidade por meio do diagnóstico e tratamento precoce dos casos humanos, e também pela diminuição dos riscos de transmissão através de controle da população de reservatórios e do agente etiológico (BRASIL, 2014).

Ressalta-se que existem poucos estudos brasileiros especificamente delineados para avaliar, conjuntamente, a efetividade do controle vetorial e da eliminação canina na redução da incidência de infecção humana (WERNECK GL, et al., 2008). Dessa maneira, um método viável seria a análise dos indicadores epidemiológicos que, de forma indireta, são capazes de demonstrar e medir a eficiência das ações.

Nessa perspectiva, o Ministério da Saúde propõe aos municípios transmissores de LV a construção e análise periódica de indicadores epidemiológicos, entomológicos e operacionais, com a finalidade de avaliar a efetividade das medidas de controle e a situação epidemiológica (BRASIL, 2014).

Um conjunto de indicadores é instrumento valioso para a gestão avaliar a situação de saúde em todos os níveis. Além disso, destina-se também a produzir evidências sobre a situação sanitária e suas tendências como base empírica para identificar grupos humanos com maiores necessidades, estratificar o risco epidemiológico, identificar áreas críticas, além de facilitar o monitoramento de objetivos e metas em saúde, estimulando a capacidade de resposta das ações e serviços (BRASIL, 2013).

Parnaíba é a segunda maior cidade do Estado do Piauí, situada na região norte do estado, com uma população de 145.705 habitantes. No período de 2001 até 2014, o Departamento de Informática do Sistema Único de Saúde (DATASUS) registou 144 casos de LV em humanos. Mesmo com um número de casos considerável, nunca no município foi realizado um estudo desse agravo que avaliasse, de fato, sua magnitude e transcendência ou as medidas de controle realizadas por intermédio da vigilância epidemiológica e ambiental. 
Nesse contexto, este estudo teve como objetivo analisar a vigilância epidemiológica da Leishmaniose Visceral em Parnaíba, durante o período de 2010 a 2014, através da construção e análise de indicadores epidemiológicos e operacionais, e também avaliar os fatores ambientais associados à ocorrência de LV.

\section{MÉTODOS}

O cenário de estudo foi o município de Parnaíba, cidade litorânea, turística e sede de macrorregião de saúde, sendo referência para onze municípios vizinhos e para os municípios de estados fronteiriços, como Maranhão e Ceará, com grande fluxo migratório de pessoas e animais.

Foi realizada uma pesquisa retrospectiva e epidemiológica para os dados coletados em fontes secundárias (SINAN, SIM e IBGE) e de coorte transversal do tipo aplicada e descritiva para os dados de fontes primárias (questionários semiestruturados, elaborados pelos pesquisadores).

Foram incluídos no estudo os novos casos humanos notificados com confirmação diagnóstica laboratorial ou clínico-epidemiológica para LV residentes e autóctones em Parnaíba, e os casos caninos com sorologia positiva para LV, ambos durante o período de 2010 a 2014.

A pesquisa foi realizada em conformidade com a resolução 466/12 do Conselho Nacional de Saúde. $O$ estudo foi aprovado pelo Comitê de Ética em Pesquisa da Universidade Federal do Piauí, campus Ministro Reis Velloso, parecer no 1.720 .009$.

Foram elaborados e analisados os indicadores epidemiológicos e operacionais padronizados pelo Programa Nacional de Controle da LV, e aplicado um questionário semiestruturado para a análise dos fatores ambientais relacionados com a LV. Para isso, foi utilizado como fonte de coleta de dados o Sistema de Informação de Agravos de Notificação (SINAN), o Sistema de Informação sobre Mortalidade (SIM), o Instituto Brasileiro de Geografia e Estatística (IBGE), a ficha de investigação epidemiológica da LV do SINAN, a planilha do resultado do inquérito canino do Centro de Controle de Zoonoses (CCZ) e um questionário semiestruturado.

As variáveis selecionadas da ficha de investigação do SINAN foram: número de casos novos autóctones de LV; número de casos confirmados por via laboratorial, clínica e epidemiológica; sexo; idade; bairro de residência; coinfecção com HIV; diagnóstico parasitológico; diagnóstico imunológico; e evolução do caso.

No SIM, foram solicitados dados referentes aos óbitos ocorridos por LV de pacientes residentes de Parnaíba no período de 2010 a 2014, e foi realizado o cruzamento das informações com o SINAN para avaliar a ocorrência de sub-registro. Os dados do IBGE foram utilizados para fazer o levantamento das características populacionais, tendo como referência o último censo de 2010.

A soro-prevalência canina foi o resultado do inquérito sorológico realizado pelo Centro de Controle de Zoonoses (CCZ) local nos últimos cinco anos, através do qual foi analisado o indicador operacional de índice de positividade canina ( $\mathrm{n}^{\circ}$ de cães sororreagentes $/ \mathrm{n}^{\circ}$ total de cães examinados $\times 100$ ).

Os indicadores epidemiológicos e operacionais foram construídos conforme os métodos de cálculos padronizados pelo Ministério da Saúde. Os resultados foram apresentados de forma descritiva de acordo com a frequência de aparecimento.

Foi aplicado um questionário ao paciente ou familiar, buscando identificar a presença e saúde dos cães, e a existência no domicílio ou peridomicílio de cenários produtores de materiais orgânicos como galinheiro, chiqueiro e lixo acumulado.

Todos os dados foram avaliados separadamente em cada variável, utilizando-se como parâmetro as diretrizes nacionais do programa de controle da Leishmaniose Visceral estabelecidas pelo Ministério da Saúde e as pesquisas científicas nacionais e internacionais.

\section{RESULTADOS}

No período de 2010 a 2014, o município de Parnaíba notificou 84 casos de LV, sendo 52 (61\%) o número de casos novos de residentes de Parnaíba; destes, 45 (53,3\%) eram casos novos autóctones. Foram analisados os 45 casos novos de LV autóctones, dos quais foram entrevistados apenas 28 pacientes $(62,2 \%)$ 
para a análise dos dados primários, pois $17(37,8 \%)$ não mais residiam no endereço da ficha de registro. A população canina foi composta por 2.787 cães constantes no inquérito sorológico canino.

\section{Indicadores epidemiológicos}

No período de 2010 a 2014, a taxa média de incidência da LV no município foi de 6,17/100.000 hab., obtendo variação entre 3,4 a 9,6 casos/100.000 hab., com maiores picos nos anos de $2011(9,6 / 100.000)$ e 2014 (8,9/100.000). A média de casos nos últimos cinco anos (2010 a 2014) foi de 9 casos, o que classifica Parnaíba como área de transmissão intensa (média de casos > 4,4) (Figura 1).

Figura 1 - Número de casos confirmados e Coeficiente de Incidência/100.000 habitantes da Leishmaniose Visceral Humana, Parnaíba-PI, Brasil, 2010-2014

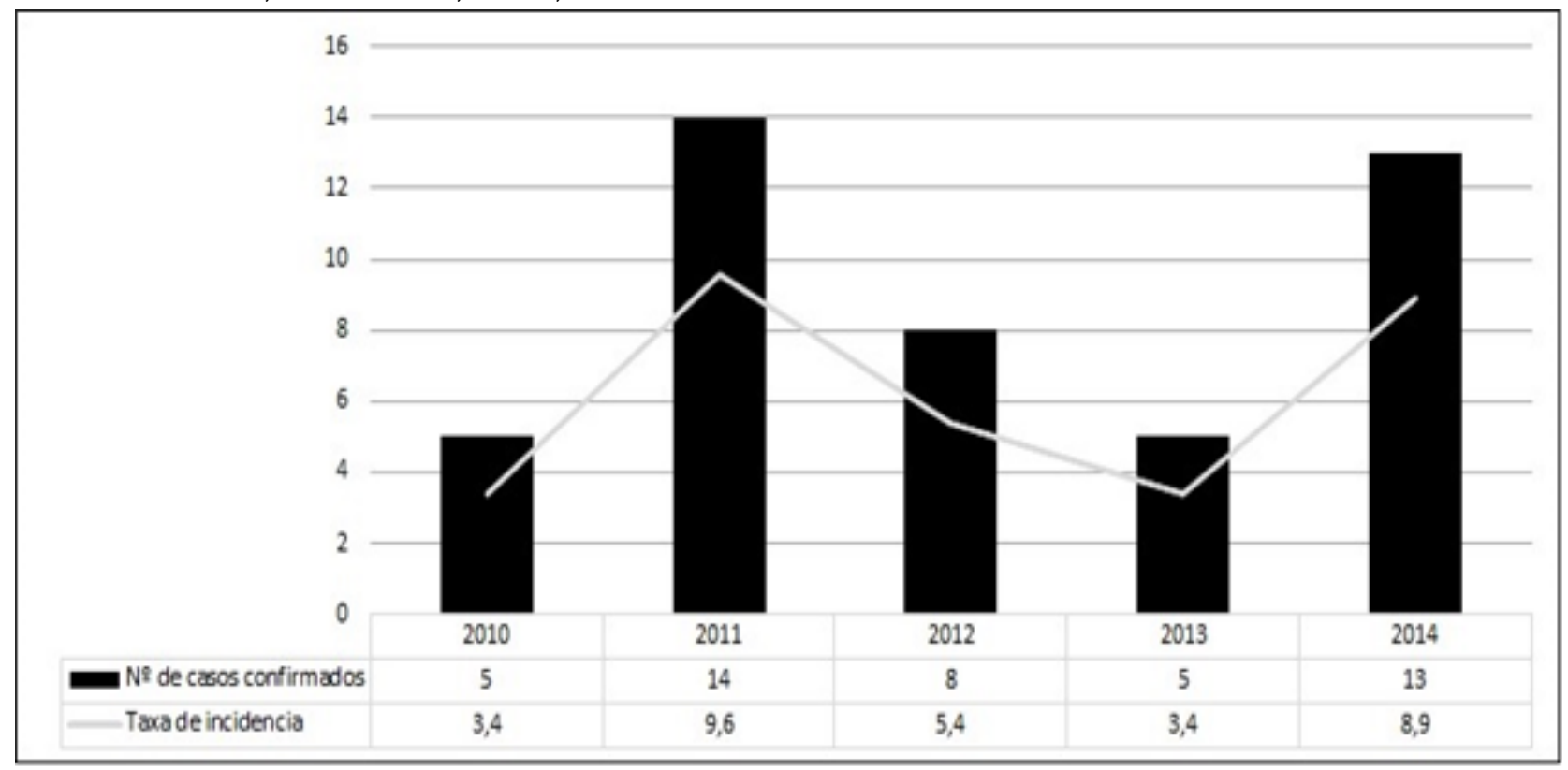

Fonte: Vigilância Epidemiológica/SINAN, Parnaíba-PI, Brasil, 2015. Adaptado.

$\mathrm{Na}$ identificação das áreas de transmissão, dos 34 bairros existentes no município, 19 apresentaram pelo menos um caso de LV, e 56\% dos bairros foram classificados como áreas de transmissão para esse agravo, o que demonstra uma ampla distribuição geográfica. Foram observadas a inclusão de novos bairros a cada ano e a continuidade de transmissão em alguns deles, como os bairros Piauí e Planalto.

A maior prevalência dos casos de LV, segundo a zona de residência, concentrou-se em áreas periurbanas (62\%), seguida da área rural (20\%) e urbana (18\%), sendo o bairro Piauí $(22 \%)$ e Planalto (20\%) aqueles com maior concentração de casos.

A taxa de letalidade no período do estudo foi de 4,4\% (n=2), com um óbito em 2011 (taxa de 7,1\%), e outro em 2012 (taxa de 12,5\%). Nos demais anos não houve registro de óbito. Os dois óbitos ocorridos foram de duas crianças (um e 10 anos), uma do sexo masculino e a outra do sexo feminino.

Em relação à faixa etária e sexo (Figura 2), a doença foi mais frequente em menores de dez anos, sendo a maior prevalência registrada na faixa entre um e quatro anos, com $31,1 \%(n=14)$, seguida da faixa etária de 20 a 39 anos, que concentrou $24,5 \%(n=11)$ dos casos. O sexo masculino foi proporcionalmente o mais acometido, com $60 \%$ ( $n=27)$, sendo que todos os indivíduos na faixa mais afetada (20 a 59 anos) eram do sexo masculino. 
Figura 2 - Distribuição dos casos, segundo faixa etária e sexo, Parnaíba-PI, Brasil, 2010-2014

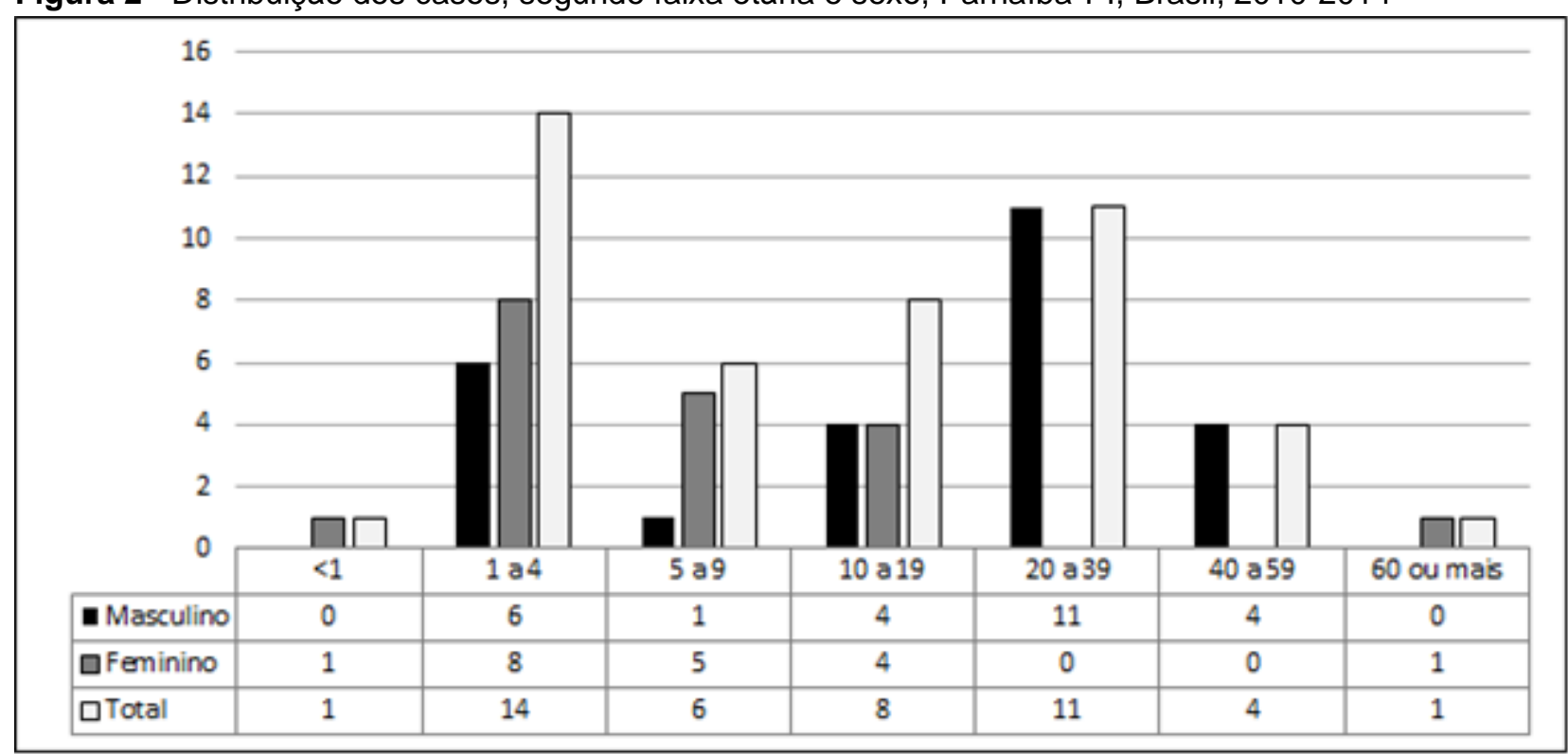

Fonte: Vigilância Epidemiológica/SINAN, Parnaíba-PI, Brasil, 2015. Adaptado.

A variável que avaliou a coinfecção HIV/LV apresentou situação ignorada em 48,9\% ( $n=22)$ dos casos, tendo sido identificado apenas 2,2\% ( $n=1)$ para a coinfecção e 48,9\% ( $n=22)$ de casos negativos, o que aponta que a coinfecção dos casos analisados pode ter sido subnotifida.

\section{Indicadores operacionais}

Os indicadores operacionais analisados neste estudo foram: a proporção de casos de LV com diagnóstico laboratorial, a proporção de casos de LV com cura clínica, e o índice de positividade canina. Sobre o índice de positividade canina, foram realizados, retrospectivamente, 2.787 testes sorológicos, com positividade presente em $85,2 \%(n=29)$ dos bairros. A soroprevalência média para Leishmaniose Visceral Canina no município foi de $16 \%$ ( $n=454)$, com variação entre $8 \%$ a $44 \%$.

Quando analisados isoladamente os anos do estudo, observou-se uma prevalência em crescente expansão, principalmente nos últimos dois anos (2013 e 2014), com um incremento de cinco vezes, quando comparado com o ano de 2014 (42\%) e 2012 (8\%). Esse incremento pode estar relacionado com a mudança do método diagnóstico da LVC adotado pelo Ministério da Saúde ocorrido no município a partir de 2013.

$\mathrm{Na}$ análise da quantidade de amostras caninas por ano, observou-se decréscimo, passando de 863 (2011) e 834 (2012) para 243 (2013) e 414 (2014). Embora o número de amostras tenha sido maior em 2011 e 2012, a prevalência foi inferior quando comparada com aquela dos anos em que o número de amostras foi maior, reforçando a efetividade do novo método diagnóstico da LVC implantado pelo Ministério da Saúde, que utiliza o teste rápido imunocromatográfico rk36 e rk39 como exame de triagem e o ELISA como confirmatório.

\section{Fatores ambientais associados à ocorrência de LV}

Dos 28 entrevistados, em $86 \%$ ( $n=24)$ foi constatada a presença de cão seja no próprio domicílio seja na residência do vizinho.

Sobre o histórico de saúde dos cães, $62,5 \%(n=15)$ dos entrevistados relataram a presença de animais suspeitos para LVC (emagrecimento, queda de pelo na região dos olhos, secreção ocular, onicogrifose, lesão de pele), 8,5\% ( $n=2)$ negaram a presença de animal suspeito e $29,2 \%(n=7)$ não souberam informar.

Em relação aos cenários produtores de matérias orgânicas, a maioria dos pacientes relatou ter convivido com um cenário que propiciou a presença do vetor $L$. longipalpis. O galinheiro concentrou $57 \%(n=16)$, seguido do lixo e/ou outros materiais orgânicos com $28,6 \%(n=8)$, o chiqueiro com $7,1 \%(n=2)$, e 7,1\% ( $n=$ 2) não tiveram contato com esse tipo de cenário. 


\section{DISCUSSÃO}

A taxa de incidência apresentou um padrão endêmico desse agravo no município, com flutuações cíclicas em intervalos regulares e inclinação de pico a cada dois anos, porém, para explicar esse comportamento cíclico epidemiológico na população de Parnaíba, faz-se necessário avaliar uma série histórica mais ampla. Desse modo, foi realizada a busca das taxas nos anos anteriores (2007: 11,3/100.000 hab.; 2008: 9,9/100.000 hab.; 2009: 2,9/100.000 hab.), sendo que o início do pico verificado a cada dois anos deu-se a partir de 2008. Durante o quinquênio, o coeficiente de incidência manteve-se maior do que a média anual brasileira (2,0 casos/100.000 hab).

Um dos fatores relacionados com a manutenção de incidências de casos de LV seria a inefetividade das ações de controle através de um sistema de vigilância permanente. $O$ que contribui pra tal fato seria a deficiência de recursos humanos e financeiros, como também uma crescente resistência social referente à eutanásia canina o que torna limitador a sustentabilidade e a cobertura no controle desse agravo. Além de outras dificuldades operacionais que somam a esse contexto como, pouca prioridade da LV frente a outras doenças; a relativa efetividade dos testes diagnósticos para a detecção da infecção canina; interferência judicial em resposta à atuação de organizações não governamentais e de clínicos veterinários contra a eutanásia; o baixo impacto das ações de educação e a precariedade das condições de saneamento ambiental (WERNECK GL, 2016).

Sobre as áreas de transmissão identificadas, os dados indicam a necessidade de se fortalecerem as medidas de controle, principalmente nos bairros mais populosos, onde a incidência foi maior. Essas ações seriam evidentemente efetivas, uma vez que as atividades nesses bairros passariam a ser mais estratégicas e pontuais, em consonância com a atual estratégia do Programa Brasileiro de Controle da LV de fortalecer as ações em áreas de transmissão (BRASIL, 2017).

O estudo revelou a periurbanização e urbanização da Leishmaniose Visceral em Parnaíba, confirmando o que vem ocorrendo no Brasil, onde o vetor encontra-se em franca expansão e adaptação em áreas urbanas e periurbanas. Esse fator pode estar fortemente relacionado com o processo de urbanização da cidade de Parnaíba, do qual fazem parte esses bairros.

Os bairros com maior incidência de casos são fronteiriços e periféricos; juntos, eles são os mais populosos do município e possuem áreas em condições sociais e econômicas precárias. Esses fatores são determinantes para a proliferação e manutenção do vetor Lutzomyia longipalpis, que tem fácil adaptação a áreas peridomésticas de zonas urbanas com condições sanitárias escassas (SANTOS, W. S. et al., 2019).

Todo esse cenário corrobora a tese de Ready D (2014), de que o ambiente propício para focos de LV são aqueles de baixo nível socioeconômico, pobreza e desnutrição na periferia das grandes cidades. Outro fator determinante, também crucial para a extensão em áreas urbanas, é a expansão urbana para áreas recémflorestadas, levando a população ao contato com os vetores por meio da invasão em seu habitat.

O indicador da taxa de letalidade de Parnaíba no período do estudo (2010 - 2014) seguiu a mesma tendência nacional, porém, no ano de 2012, observou-se uma taxa mais elevada, podendo estar relacionada com a fragilidade na qualidade assistencial. É valido ressaltar que foi identificado um óbito subnotificado no SINAN, despertando a possibilidade da subnotificação de óbitos por LV.

A letalidade no país vem aumentando gradativamente nos últimos anos, com uma taxa de $7,2 \%$ no período de 2003 a 2018. No último boletim epidemiológico sobre taxa de letalidade em 2013, a região Nordeste registrou 5,5\% e Piauí, 4,7\% (BRASIL, 2019).

$\mathrm{Na}$ análise dos dados primários, para o caso que veio a óbito em 2012, foi verificado um tempo de diagnóstico maior que dois meses, com mais de quatro procuras aos serviços de saúde, com sinais e sintomas, no final da doença, de desnutrição e anemia severas, quadro infeccioso, icterícia, astenia e fenômenos hemorrágicos, fatores determinantes para um pior prognóstico.

Assim, pode-se inferir que houve demora no diagnóstico, configurando uma situação de alerta emergencial. Reforçando isso, o estudo identificou também que $77 \%(n=35)$ dos diagnósticos ocorreram na 
rede hospitalar, $15,6 \%(n=7)$ na atenção básica e 6,7\% $(n=3)$ no ambulatório. Esses dados evidenciam a ocorrência de diagnóstico tardio, uma vez que a porta de entrada do usuário no serviço de saúde deve ser a atenção básica. Esses dados reforçam a necessidade de se fortalecer a capacidade de resposta desses serviços para um diagnóstico e tratamento precoces (BRASIL, 2017).

Com relação a faixa etária e sexo, o resultado do estudo não seguiu a mesma tendência nacional. Nesta pesquisa, os adultos jovens foram os mais afetados enquanto em outros estudos os mais acometidos foram crianças e idosos.

As crianças menores de 10 anos constituem o grupo mais susceptível devido à imaturidade do sistema imune, agravada pela desnutrição associada à baixa condição socioeconômica, tão comum em áreas endêmicas, e ainda pelo fato de as crianças estarem mais expostas ao flebotomíneo no ambiente peridomiciliar (BRASIL, 2017; ALMEIDA AS, 2011). No entanto, quando o adulto jovem é acometido, pode-se suspeitar de comprometimento do sistema imunológico, que pode ser ocasionado por vários agravos, entre eles o vírus da imunodeficiência humana / síndrome da imunodeficiência adquirida (HIV/AIDS) e o uso de álcool e drogas, entre outros que atingem o sistema imunológico.

Esses achados também foram identificados após a aplicação do questionário, despertando a atenção para o relato das mães dos pacientes sobre a dependência de drogas ilícitas, e revelando uma proporção de $22 \%$ $(n=5)$ dos pacientes do sexo masculino na faixa entre 10 a 49 anos com dependência química. Sabe-se que esse é um dos grandes problemas atuais da saúde pública, deixando esse grupo em situação de vulnerabilidade para muitas doenças negligenciadas. A coinfecção HIV/LV é um problema em expansão, da mesma forma que se verifica há muito tempo a crescente associação entre HIV/AIDS e os usuários de drogas injetáveis. Por isso, torna-se imperioso fortalecer a vigilância da coinfecção LV/HIV por meio da garantia da oferta da testagem para HIV em todos os pacientes com Leishmaniose Visceral.

Alguns estudos relatam o aumento do número de casos da LV em pacientes imunodeprimidos (ex.: pessoas com AIDS) como um dos fatores responsáveis pelo incremento da elevação global desse agravo (MONTALVO AM, et al., 2012). Anteriormente ao aparecimento da Aids no Mediterrâneo, a LV era uma doença que tradicionalmente afetava crianças. Entretanto, a doença passou a acometer principalmente adultos entre 31 e 50 anos de idade, ocorrendo na forma de coinfecção em $70 \%$ dos casos (CHICHARRO C, et al., 2020).

O indicador operacional de índice de positividade canina apontou ampla distribuição geográfica da LVC no município estudado, apresentando uma razão de 10:1 em relação aos casos humanos. Pode-se inferir que há áreas silenciosas para LV humana uma vez que a infecção canina precede a humana; além disso, alguns estudos relacionam a incidência da doença em humanos com a ocorrência de LV canina (Brasil, 2014).

Os resultados sobre a presença e saúde dos cães mostraram uma estreita relação entre o reservatório e o hospedeiro, tornando-se ainda mais forte quando há animal suspeito. Tal resultado corrobora vários estudos que relatam a correlação espacial da doença em humanos e a alta prevalência da infecção canina, sugerindo que, na presença do flebotomíneo, a LVC é um fator chave no desencadeamento da transmissão em seres humanos (SANTOS WS, et al., 2019).

É válido destacar neste estudo que $62,5 \%$ dos cães presentes no domicílio dos pacientes ou no dos seus vizinhos no período da doença eram suspeitos para LVC. Esse dado reforça a importância do cão na cadeia de transmissão desse agravo, mais ainda quando há cão suspeito, principalmente domiciliado. Vários estudos apontam a relevância apenas de cães sintomáticos na transmissibilidade da doença, porém outros defendem que cães assintomáticos também apresentam riscos de transmissão, embora menor que os sintomáticos (COSTA DNCC et al., 2018).

É essa base teórica que sustenta as ações de controle canino do Programa Nacional da Leishmaniose, partindo do ponto de que a incidência da infecção humana está diretamente relacionada com o número de cães infectados. Segundo o Instituto Pasteur (2013), a razão entre a população canina e a população humana varia de 1:6 a 1:10 (INSTITUTO PASTEUR, 2013). 
O resultado deste estudo condiz com um estudo realizado no Estado do Pará por Santos WS et al. (2019), onde seus resultados afirmam que galinheiros, com as aves domésticas, mostraram-se como os ecótopos mais atrativos para os vetores, devido ao elevado número de espécies que foi registrado nesse tipo de estrutura artificial principalmente no período chuvoso, indicando uma possível associação entre os insetos e as aves domésticas (SANTOS WS et al., 2019).

O cenário rico em matéria orgânica gerado por animais domésticos e más condições sanitárias, além do solo exposto e da vegetação com raízes e tronco de árvores, é propício à manutenção e proliferação do Lutzomyia longipalpis (PIRAJÁ GV e LUCHEIS SG, 2014). Assim, o estudo mostra a possibilidade de esses cenários serem fatores determinantes ativos na manutenção da incidência de LV no município.

É importante enfatizar a relevância dos chiqueiros e galinheiros como locais de criação e manutenção de alta densidade de flebotomíneos no ambiente, constituindo-se um fator de risco que deve ser considerado na vigilância epidemiológica para o planejamento de ações de controle das leishmanioses (MARQUES, R. A. 2019).

Esse processo é facilitado, ainda, pelo hábito que as pessoas têm de manter os quintais com seu aspecto rústico, com solo exposto, arborizado e associado com abrigos de animais e acúmulo de materiais diversos, especialmente matéria orgânica, criando um ambiente favorável para a procriação e 0 abrigo dos flebotomíneos (LARA-SILVA FO, et al., 2015).

\section{CONSIDERAÇÕES FINAIS}

Nossos resultados sugerem que as medidas de controle ambientais são importantes para o controle da LV, pois conhecendo-se o nicho onde o vetor se desenvolve, as autoridades de saúde locais poderão traçar estratégias focadas com grande possibilidade de impacto nas medidas de vigilância e controle da doença. $O$ conjunto dos indicadores apresentados produziram informações sobre a magnitude e transcendência da Leishmaniose Visceral em Parnaíba, identificaram ações que precisam ser fortalecidas, como também buscaram relacionar os fatores ambientais com a ocorrência da LV. Portanto, os resultados alcançados nesta pesquisa podem servir como instrumento de planejamento estratégico no controle da LV, focalizando as intervenções em áreas consideradas de risco e, dessa forma, incrementando a efetividade das medidas de controle. Essas medidas poderiam levar a uma redução da incidência de casos de LV em humanos a longo prazo.

\section{REFERÊNCIAS}

1. ALMEIDA AS. Identificação de áreas sob maior risco para leishmaniose visceral, na cidade de Teresina, Piauí, Brasil [tese de doutorado]. Rio de Janeiro: Instituto de Medicina Social da Universidade Federal do Rio de Janeiro, 2011.

2. BRASIL. Ministério da Saúde. Secretaria de Gestão Estratégica e Participativa. Departamento de Articulação Interfederativa. Caderno de Diretrizes, Objetivos, Metas e Indicadores: 2013 - 2015. 1. Brasília: Ministério da Saúde, 2013.

3. BRASIL. Ministério da Saúde. Leishmaniose visceral: o que é, causas, sintomas, tratamento, diagnóstico e prevenção [Internet]. Brasília: Ministério da Saúde, 2016.

4. BRASIL. Ministério da Saúde. Secretaria de Vigilância em Saúde. Coordenação-Geral de Desenvolvimento da Epidemiologia em Serviços. Guia de vigilância em saúde: volume 3 [Internet]. Brasília: Ministério da Saúde, 2017.

5. BRASIL. Ministério da Saúde (BR). Secretaria de Vigilância em Saúde. Vigilância em saúde no Brasil 2003|2019: da criação da Secretaria de Vigilância em Saúde aos dias atuais. Bol Epidemiol [Internet]. 2019 set [data da citação]; 50 (n.esp.):1-154.

6. COSTA DNC, et al. Culling Dogs in Scenarios of Imperfect Control: Realistic Impact on the Prevalence of Canine Visceral Leishmaniasis. PLoS One Negl Trop 2013; 7(8): e2355

7. Costa DNC et al. Leishmaniose visceral em humanos e relação com medidas de controle vetorial e canino. Rev Saúde Pública 2018; 52(92): 1-11.

8. INSTITUTO PASTEUR. Nota técnica 02 - IP/CCD/SES-SP - Secretaria de Estado de Saúde do Estado de São Paulo - Coordenadoria de Controle de Doenças, 2013.

9. LARA-SILVA FO, et al. Epidemiological aspects of vector, parasite, and domestic reservoir in areas of recent transmission and no reported human cases of visceral leishmaniasis in Brazil. Acta Trop 2015; 148: 128-36.

10. LEITE BMM et al. The mass use of deltamethrin collars to control and prevent canine visceral leishmaniasis: a field effectiveness study in a highly endemic area. PLoS Negl Trop Dis 2018; 12(5): E0006496. 
11. MARQUES RA. Fauna flebotomínica em uma área endêmica para leishmaniose visceral. 2019 . 35 f. Trabalho de Conclusão de Curso (Bacharelado em Medicina Veterinária) - Unidade Acadêmica de Garanhuns, Universidade Federal Rural de Pernambuco, Garanhuns, 2019.

12. MONTALVO AM, et al. Diagnóstico de la leishmaniasis: de la observación microscópica del parásito a la detección Del ADN. Rev Cub Med Trop 2012; 63(2): 108-31.

13. PIRAJÁ GV, LUCHEIS SG. A vigilância epidemiológica de flebotomíneos no planejamento de ações de controle nas leishmanioses. Vet Zootec 2014; 21(4): 503-15.

14. READY PD. Epidemiology of Visceral Leishmaniasis. Clin Epidemiol 2014; 3(6): 147-54.

15. SILVA FS, et al. Flebotomíneos (Díptera: Psychodidae) associados a abrigos de animais domésticos em área urbana do nordeste do estado do Maranhão, Brasil. Rev Patol Trop 2012; 1(3): 333-47.

16. SANTOS WS et al. Flebotomíneos (Psychodidae: Phlebotominae) de área endêmica para leishmaniose cutânea e visceral no nordeste do estado do Pará, Brasil. Rev Pan-Amaz Saúde 2019; 10: 1-8.

17. WERNECK GL, et al. Avaliação da efetividade das estratégias de controle da leishmaniose visceral na cidade de Teresina, Estado do Piauí, Brasil: resultados do inquérito inicial - 2004. Epidemiol Serv Saúde 2008; 78(2): 87-96.

18. WERNECK GL. Controle da leishmaniose visceral no Brasil: o fim de um ciclo? Cad. Saúde Pública 2016; 32(6): eED010616.

19. WORLD HEALTH ORGANIZATION. Leishmaniasis: factsheet. Geneva, 2016. (WHO - Media Centre, 375). 